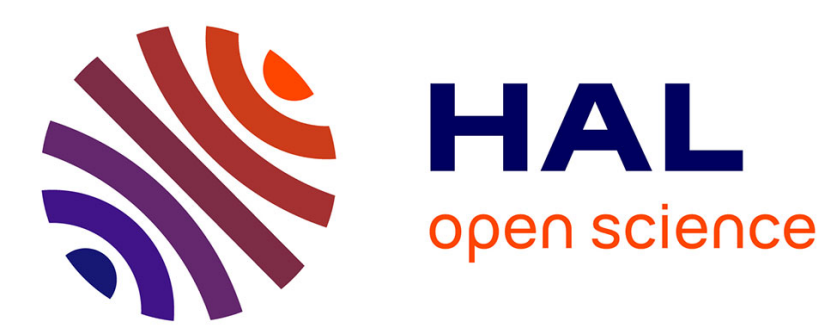

\title{
Les littoraux à Zanzibar : des espaces investis et subvertis par le tourisme \\ Pascal Villecroix
}

\section{To cite this version:}

Pascal Villecroix. Les littoraux à Zanzibar: des espaces investis et subvertis par le tourisme. Expressions, 1999, Spécial Histoire-Géographie, 13, pp.69-89. hal-02406144

\section{HAL Id: hal-02406144 \\ https://hal.univ-reunion.fr/hal-02406144}

Submitted on 12 Dec 2019

HAL is a multi-disciplinary open access archive for the deposit and dissemination of scientific research documents, whether they are published or not. The documents may come from teaching and research institutions in France or abroad, or from public or private research centers.
L'archive ouverte pluridisciplinaire HAL, est destinée au dépôt et à la diffusion de documents scientifiques de niveau recherche, publiés ou non, émanant des établissements d'enseignement et de recherche français ou étrangers, des laboratoires publics ou privés. 


\title{
LES LITTORAUX À ZANZIBAR : DES ESPACES INVESTIS ET SUBVERTIS \\ PAR LE TOURISME
}

\author{
Pascal VILLECROIX \\ IUFM de la Réunion
}

\begin{abstract}
7 anzibar, petit archipel côtier au nord du canal du Mozambique, composé de deux îles, Unguja et Pemba, forme, avec Mafia, la Tanzanie insulaire. Plus de 90000 visiteurs ont séjourné à Zanzibar en $1998^{2}$ alors qu'au milieu des années quatre-vingts, le tourisme apparaissait comme une activité marginale de l'économie locale. C'est brutalement depuis quelques années que l'activité touristique s'est emparée de l'espace zanzibari. Les littoraux nord et est, longtemps délaissés, sont ainsi investis par le tourisme international ; la capitale, Zanzibar-ville, sur la côte ouest, est redynamisée alors qu'elle s'enfonçait dans une lente décrépitude. La plupart des paysages littoraux subissent d'exceptionnelles mutations, bien qu'ils soient occupés et parcourus différemment par les vacanciers. Ce phénomène récent réorganise le territoire de lîle. Cela se traduit à la fois par une conquête d'espaces nouveaux et par un processus de récupération-valorisation ${ }^{3}$. Cette mise en tourisme des lieux s'apparente-t-elle à une subversion spatiale ? Ce système estil commun ou atypique dans l'organisation spatiale des îles du sud-ouest de l'océan Indien ? Cette émergence des flux de loisirs touristiques réveille néanmoins cet espace et donne à son insularité une autre valeur.
\end{abstract}

L'aspect spatial du tourisme guidera cette réflexion, en mettant en exergue, dans un premier temps, les flux des voyageurs et leurs logiques d'ancrage géographique. Il faudra ensuite s'interroger sur les facteurs tant naturels qu'humains qui ont permis cette redécouverte pour enfin apprécier les nouveaux paysages de Zanzibar nés de cette explosion du tourisme. 
échelle

0
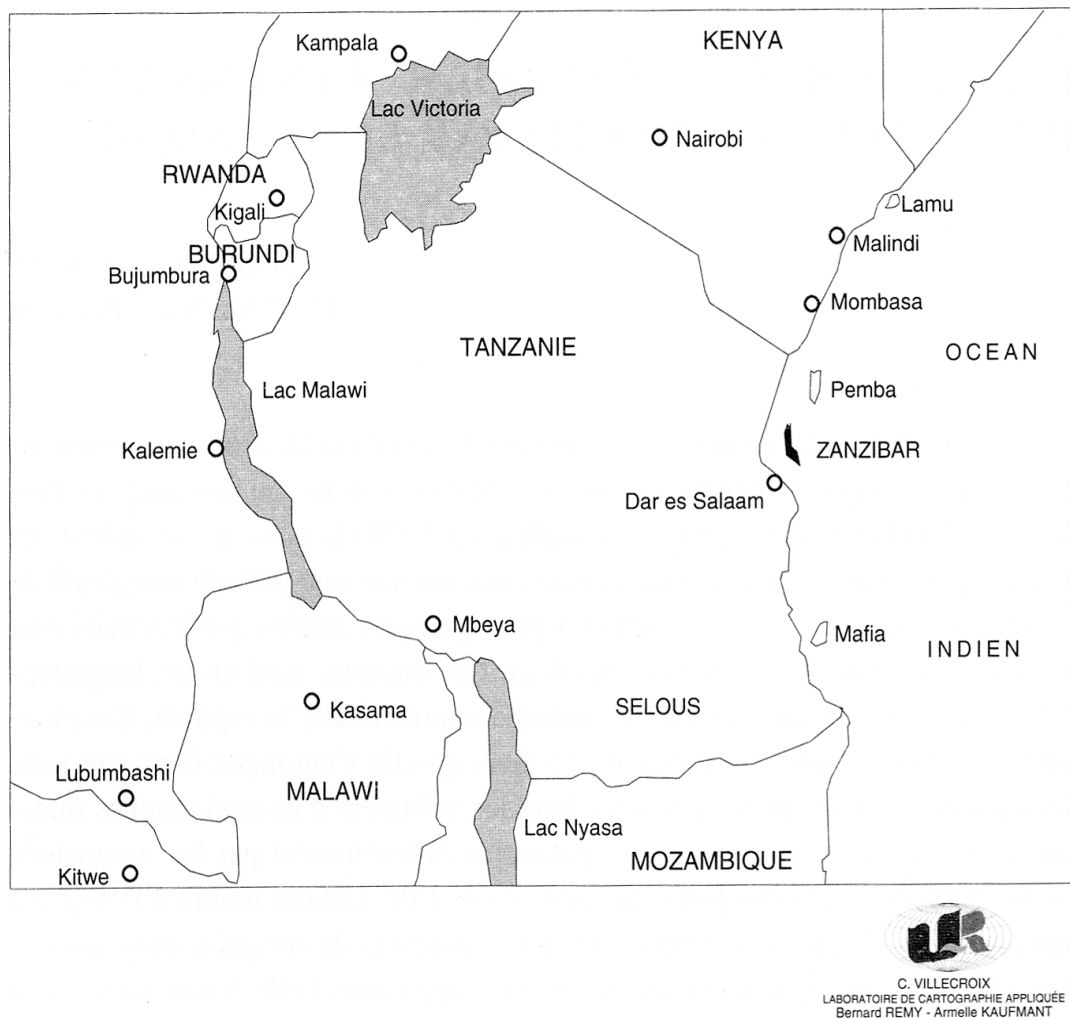

\section{Une mise en tourisme des lieux}

\subsection{Les Italiens donnent à Zanzibar une dimension touristique internationale}

De 1983 à 1997, le nombre de touristes a été multiplié par plus de huit, passant de 10000 à 86000 . Le cap des 100000 entrées devrait être atteint, selon le gouvernement, pour l'an 2000. Depuis 1997, l'île est entrée dans l'ère du tourisme de masse. Le graphique $\mathrm{n}^{\circ} 1$ souligne cette croissance spectaculaire 
et constante des visiteurs avec une vive accélération pour les deux dernières années ( $23 \%$ d'augmentation entre 1995 et 1997). Même la guerre du Golfe (1991) n'a pas entravé la progression des visiteurs. Pourtant, en 1994, une baisse soudaine et brutale entraîne une perte de 27000 entrées par rapport à l'année précédente. Cette rupture modère et tempère notre analyse globale. Cette chute peut-être imputée à une vague d'épidémies qui touche à cette époque la Tanzanie continentale et frappe par contrecoup l'essor de son tourisme insulaire. Cet exemple démontre une fois de plus l'extrême fragilité de ce secteur de l'économie surtout dans l'un des pays les moins avancés de la planète (PMA).

Graphique $n^{\circ} 1$ :

Nombre d'entrées de touristes à Zanzibar de 1986 à 1998

(source : Commission for tourism, Zanzibar)

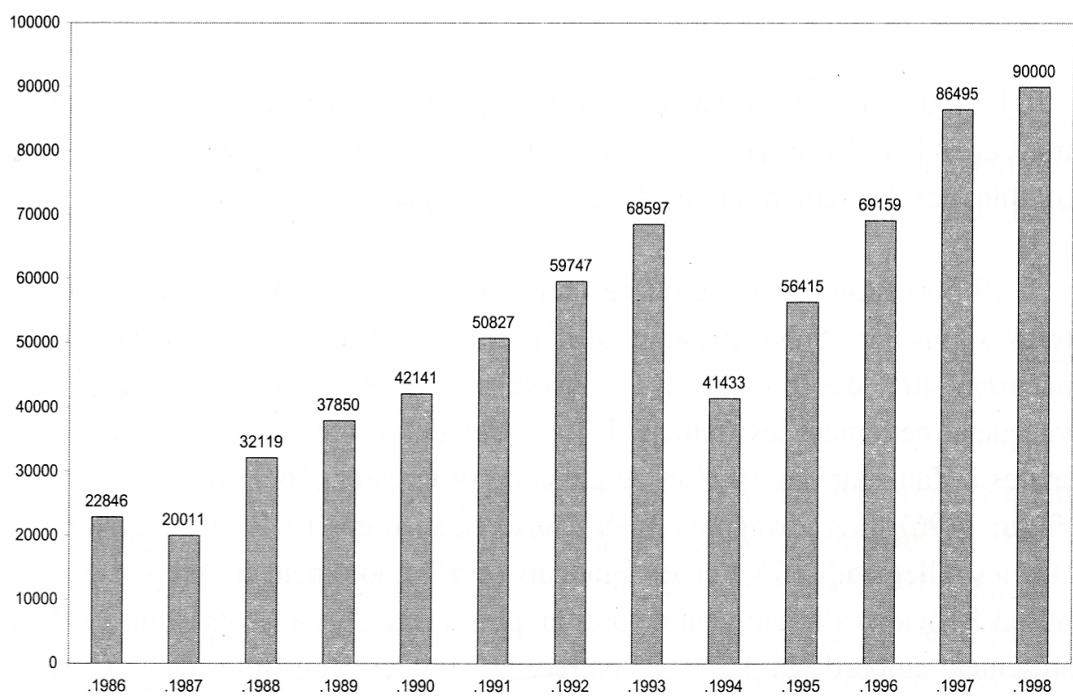

Pour mieux apprécier quantitativement cette nouvelle destination, nous devons replacer le nombre d'entrées à Zanzibar dans le contexte touristique des îles du sud-ouest de l'océan Indien ${ }^{4}$. Zanzibar se situe en cinquième position derrière l'île Maurice, la Réunion, les Seychelles et Madagascar mais devant Mayotte et les Comores (graphique $n^{\circ} 2$ ). À son rythme actuel de croissance, Zanzibar va très vite dépasser Madagascar et se rapprocher des Seychelles. 


\section{Graphique $n^{\circ} 2$ : \\ Nombre d'entrées de touristes \\ dans les îles du sud-ouest de l'océan Indien en 1997 \\ (source : INSEE-Réunion)}

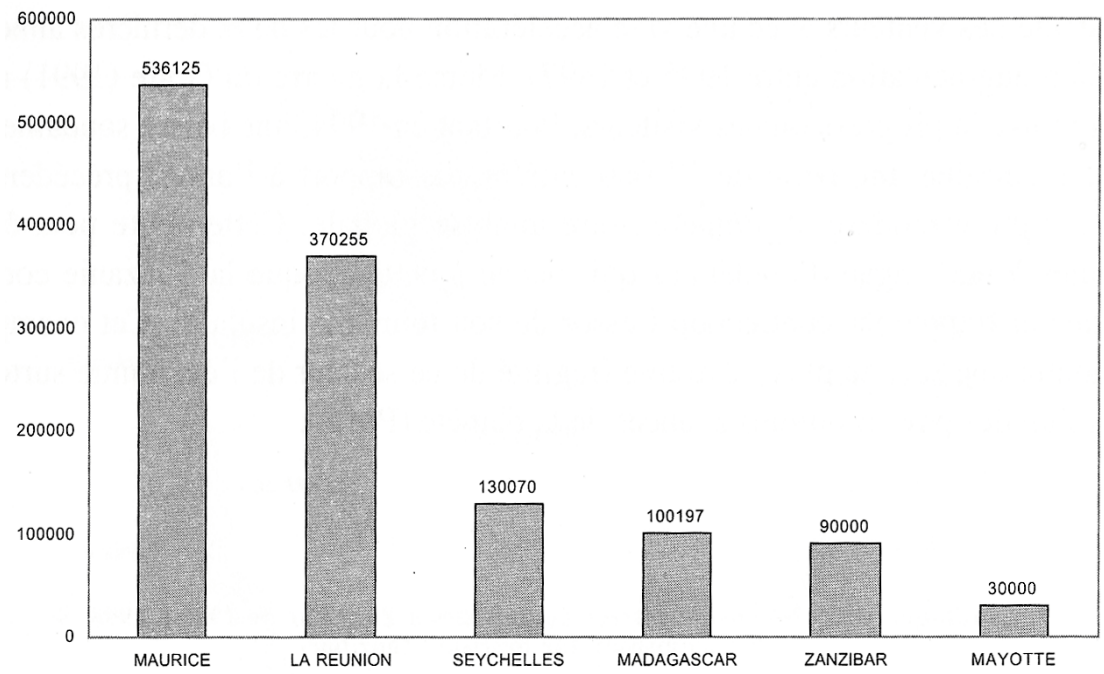

Le tourisme à Zanzibar se caractérise par l'origine internationale des visiteurs. Cependant, l'Europe est le continent le plus représenté avec une très forte prédominance des Italiens et des Anglais (graphique $\mathrm{n}^{\circ} 3$ ).

19500 Italiens ont séjourné dans l'île en 1997, soit $23 \%$ du total des entrées, suivis par 15000 Britanniques (17\%). Ces deux flux émetteurs majeurs concentrent $40 \%$ des touristes. Il y a encore six ans, les Anglais et les Allemands devançaient nettement les Italiens. L'Italie devient le premier foyer émetteur de touristes à Zanzibar dès 1993 et ne cesse de progresser (16 $\%$ du total en 1995, $19 \%$ en 1996). Les voyageurs américains et canadiens (9 $\%)$, les Scandinaves (9\%), les Allemands $(6 \%)$ et les Français $(5,5 \%)$ dominent un second groupe. Les Sud-Africains s'intéressent de plus en plus à cette île ; absents avant 1995, ils représentent aujourd'hui 3,5\% des entrées et devraient constituer un flux majeur dans les prochaines années. De nombreux projets hôteliers sud-africains sont en cours aussi bien à Zanzibar-ville que sur les littoraux nord et est. 
Graphique $n^{\circ} 3$ :

Nombre d'entrées de touristes à Zanzibar par nationalité. (source : Commission for tourism, Zanzibar)

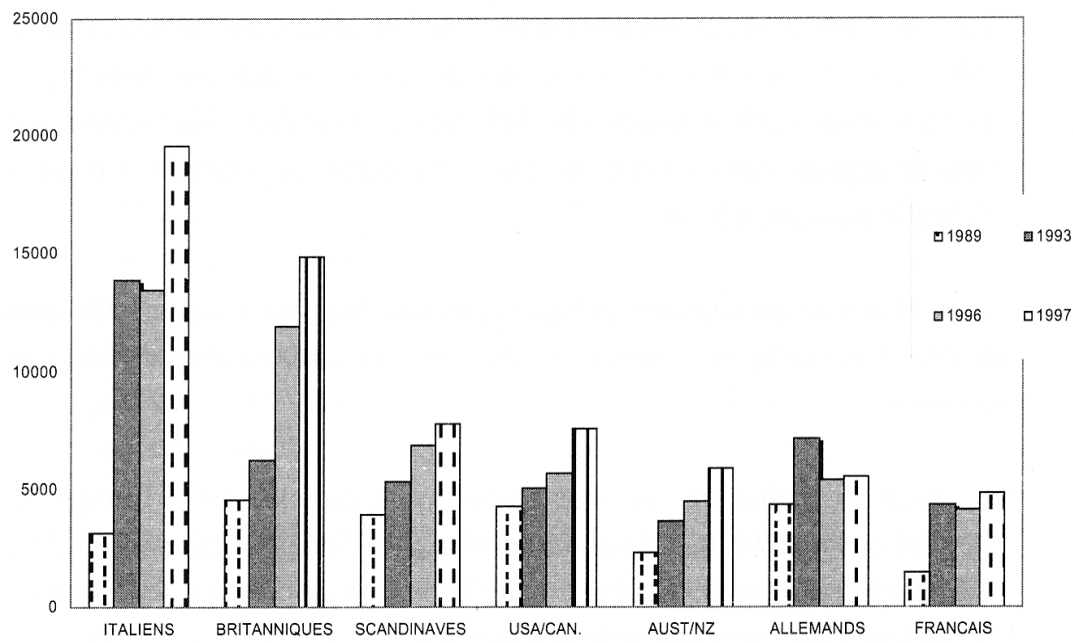

\subsection{Du tourisme d'escale au tourisme de séjour balnéaire}

Le tourisme d'escale s'imposait exclusivement dans l'île avant la venue massive des Italiens. Après un safari, les visiteurs en Tanzanie continentale ou au Kenya recherchaient avant tout les plaisirs balnéaires et l'aspect culturel de la capitale. Ils arrivaient en majorité par bateau de Mombassa (Kenya) ou de Dar-es-Salaam (Tanzanie). Ceci est encore vrai aujourd'hui, mais en partie seulement, car les Italiens ont modifié le type et les structures du tourisme à Zanzibar. La rupture qualitative est nette, elle entraînera, nous le verrons, deux logiques, deux ancrages géographiques. Plus de $90 \%$ des touristes italiens viennent directement de Milan par charters alors que les touristes britanniques restent attachés au tourisme d'escale.

Les moyens d'entrées peuvent nous aider, en partie, à dégager quatre types de touristes à Zanzibar :

- Le touriste «sac à dos» prédomine jusqu'à la fin des années quatrevingts. Le « routard » vient dans lîle le plus souvent par bateau de Dar-esSalaam ou de Mombassa. Les professionnels du tourisme estiment que ce type de tourisme représente encore 30 à $40 \%$ du total des entrées en 1998. Ce touriste rapporte peu de devises car il ne fréquente que les pensions et se 
déplace en taxi collectif (daladala). Zanzibar est une étape de trois à quatre jours dans son périple de l'Afrique de l'Est.

- Le deuxième type de touriste est plus âgé et aisé, il arrive dans l'île de préférence en avion après un safari. Il séjourne dans des hôtels plus luxueux situés dans le quartier traditionnel de Zanzibar-ville (Stonetown) mais ne néglige pas les hôtels de plage. La durée moyenne de son séjour n'excède pas quatre jours.

- Le croisiériste est atypique puisqu'il parcourt mais n'occupe pas l'espace de l'île. Les quelques heures d'escale sont consacrées à Stonetown et ses environs.

- Le touriste «balnéaire », dernier arrivé, vient directement d'Europe par charters et séjourne dans sa coquille hôtelière. Ses activités sont exclusivement centrées sur le littoral : bain, bronzage et plongée sous marine. Son séjour varie entre une à deux semaines.

Ces quatre types de touristes ont des comportements spatiaux très différents. Ils vont d'une manière ou d'une autre marquer les paysages zanzibaris.

\subsection{Types et formes d'espaces touristiques}

Depuis 1986, date à laquelle Zanzibar s'ouvre au tourisme international, nous observons distinctement un processus d'investissement de l'espace par le tourisme, avec des stades successifs classiques (appropriation foncière, installation et gestion des équipements, articulation avec le milieu local). Trois vagues de construction hôtelière se suivent :

- La première, de 1986 à 1989, marque une période de réalisation de guest-houses sur la côte est/sud-est de l'île. Ces structures d'accueil bien adaptées au style «routard » de l'époque, disposent en moyenne de 10 à 20 chambres. Nous pouvons souligner l'étroite corrélation entre les flux de fréquentation touristique d'une part, les types et formes d'espaces touristiques d'autre part.

- La deuxième vague de construction hôtelière, de 1990 à 1992, place d'emblée l'île dans le concert du tourisme international. Plusieurs hôtels-clubs se construisent sur la côte est, les capitaux sont étrangers, essentiellement italiens ; ce sont des complexes de 100 à 280 lits. Des centaines de bungalows forment ainsi un véritable ruban de 45 kilomètres sur le littoral.

- La troisième et dernière vague de construction touche, depuis 1992, Zanzibar-ville et plus précisément la vieille ville (Stonetown). La ville portuaire, qui compte près de 100000 habitants, constitue la partie la plus authentique. Ce centre ancien est d'une telle richesse qu'il a été classé en 1985 par I'UNESCO comme l'un des le sites historiques mondiaux à protéger en 
priorité. Zanzibar-ville était l'une des villes les plus décrépites de l'Afrique de l'Est. De vieilles demeures transformées en hôtels de caractère, avec des capitaux souvent locaux, contrastent avec la partie orientale de l'île. Le tourisme a en grande partie contribué à la réhabilitation de Stonetown. Cette ville hors du temps retrouve petit à petit sa splendeur perdue. Alors qu'elle souffrait d'une réelle défaveur, elle est devenue un espace de récréation.

Au total, Zanzibar compte aujourd'hui 110 hôtels (des guest-houses aux villages-clubs en passant par les hôtels de caractère) répartis dans deux espaces touristiques que tout oppose (carte $n^{\circ} 2$ ). D'un côté, un espace qui s'appuie sur le processus « conquête d'espaces nouveaux », de l'autre, un espace ancien structuré autour du processus « récupération-valorisation».

La dynamique touristique s'exprime autant par la linéarité (côte est) que par les installations ponctuelles (Stonetown). La vieille ville concentre 49 hôtels soit 44,5\% du total, Zanzibar-ville (en dehors du centre ancien) 17 hôtels soit $15,5 \%$, la côte est, 38 hôtels $(34,5 \%)$ et la côte nord, six hôtels soit $5,5 \%$.

Cette mise en tourisme des lieux a très vite mis en évidence les insuffisances et faiblesses des infrastructures de l'île en particulier le réseau de transport intérieur. Les routes bitumées sont peu nombreuses, en mauvais état et souvent impraticables pendant la saison des pluies. Seuls trois axes routiers goudronnés desservent Zanzibar : Zanzibar-ville / Nungwi (extrême nord de 1'île), Zanzibar- ville / Chwaka (côte est) et Zanzibar-ville / Pete (sud-ouest). Le reste du réseau est constitué de pistes de terre utilisables ponctuellement dans l'année. Quelques exemples de durée de trajet témoignent des difficultés et de la lenteur des déplacements ; en partant de Zanzibar-ville, il faut plus d'une heure pour joindre Chwaka, plus de deux heures pour Jambiani et près de trois heures pour Bwejuu (carte $n^{\circ} 2$ ). Malgré l'amélioration d'une partie du réseau routier desservant la côte est, le transport intérieur demeure un handicap évident.

L'aéroport de Kisauni, le seul de Zanzibar, se situe à 6 kilomètres au sud de Stonetown, la route est directe et rapide pour joindre les hôtels du centreville ; par contre, les transferts vers les hôtels des côtes nord et est restent problématiques. Le réseau routier est davantage centré sur la façade ouest de l'île, créant ainsi un déséquilibre préjudiciable aux hôtels de la côte est. Cette mise en tourisme des lieux crée donc un dysfonctionnement dans l'organisation de l'espace et ce phénomène ira en s'accentuant dans les prochaines années si des efforts ne sont pas faits pour rééquilibrer le réseau routier de l'île. 


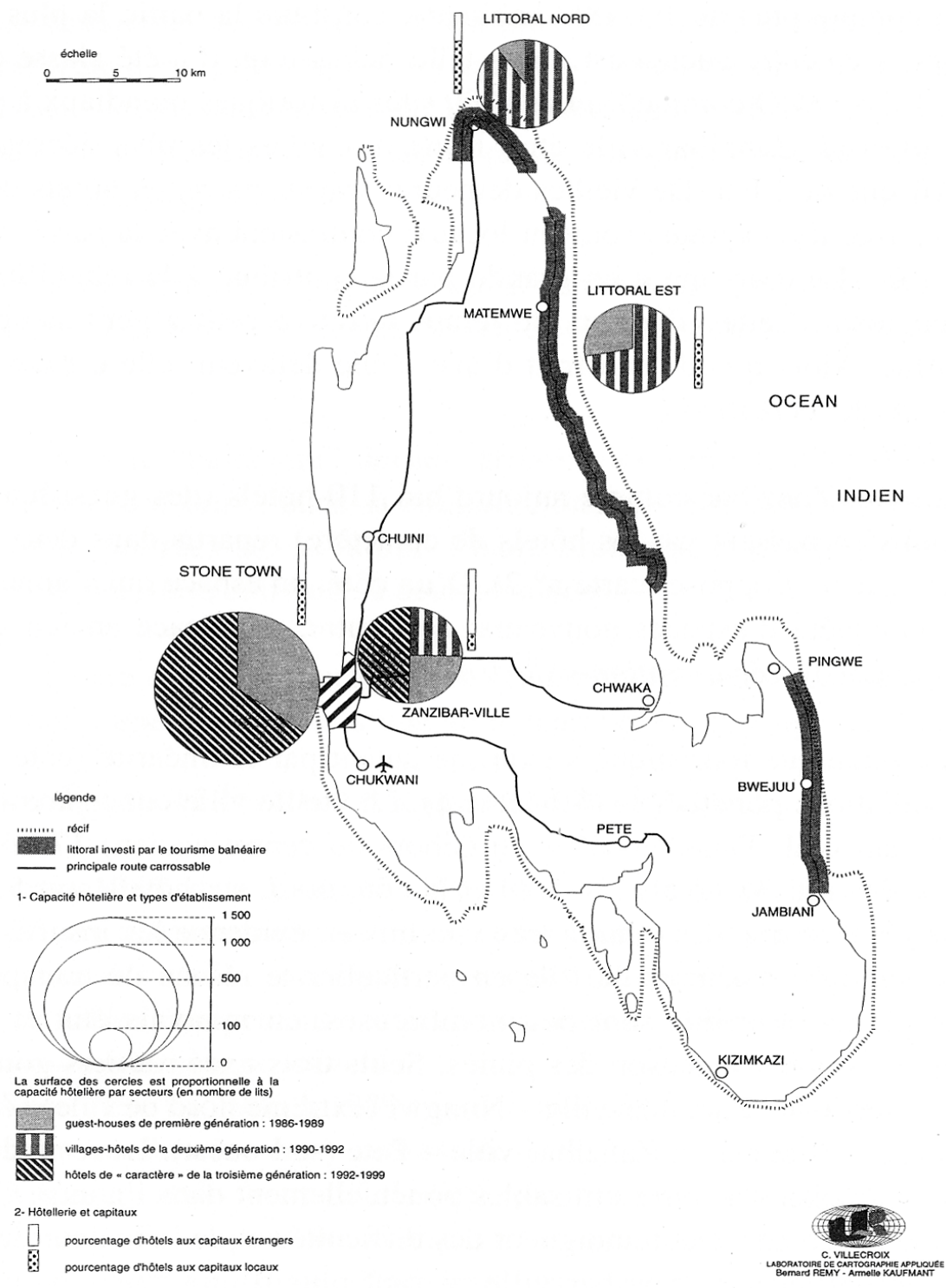

Carte $n^{\circ} 2$ :

Répartition spatiale des structures hôtelières à Zanzibar 
Les télécommunications ont fait de réels progrès depuis deux ans et facilitent tous contacts avec l'extérieur. Les tour-operators bénéficient de cette évolution, informations et réservations posent beaucoup moins de problèmes qu'autrefois.

\section{Une convergence d'éléments favorables crée la destination « Zanzibar »}

I1 n'y a pas de hasard dans le soudain et brutal boom touristique à Zanzibar depuis quelques années ; il a fallu une convergence d'éléments favorables pour créer cette nouvelle destination touristique qui a désormais une place de choix dans l'espace insulaire du sud-ouest de l'océan Indien. Les conditions naturelles, le patrimoine culturel et historique, l'ouverture économique depuis 1984, l'évolution des transports, la position de l'île par rapport aux flux touristiques de l'Afrique de l'Est, sont autant d'éléments déclencheurs de ce formidable essor.

\subsection{Climat et relief côtier : atouts pour l'extension du tourisme balnéaire}

Le binôme «climat-relief » est essentiel pour la compréhension de l'espace touristique à Zanzibar. Il explique, en partie, les flux des visiteurs mais aussi la localisation des structures d'accueil.

Souvent, le climat est un facteur pouvant jouer en faveur (héliotropisme) ou en défaveur (mousson) du tourisme. L'héliotropisme est un argument fondamental justifiant à lui seul d'importants flux de fréquentations touristiques. Les catalogues des tour-operators proposant Zanzibar mettent en évidence l'ensoleillement et le rythme climatique marqué de l'île. Les températures de l'archipel sont chaudes et stables toute l'année. L'amplitude thermique annuelle ne dépasse guère $6^{\circ} \mathrm{C}$. Août est le mois le plus frais avec $23^{\circ} \mathrm{C}$, février le plus chaud avec $29^{\circ} \mathrm{C}$. Ce ne sont pas les températures qui vont influencer les flux touristiques mais la pluviométrie ; Zanzibar n'échappe pas à cette règle du monde intertropical. Les précipitations suivent ici le rythme du déplacement de la convergence tropicale ; ainsi, le climat de l'île est caractérisé par deux saisons des pluies. La première (Masika) d'avril à mai est très marquée avec 250 à $400 \mathrm{~mm} / \mathrm{mois}$; ces pluies correspondent à un flux de sud-est ; les alizés maritimes chargés d'humidité se déchargent sur les terres qu'ils rencontrent. C'est une période creuse pour le tourisme : 3000 et 3500 
entrées pour les mois d'avril et de mai en 1997. Par contre, la petite saison des pluies (Mvuli) avec 90 à $200 \mathrm{~mm}$ par mois, d'octobre à décembre, n'a aucune incidence sur la fréquentation touristique. Lors des saisons sèches (janvier-février et juin-septembre), les précipitations mensuelles n'excèdent pas $80 \mathrm{~mm}$ (tableau $\mathrm{n}^{\circ} 1$ ). Les précipitations ne sont pas uniformément réparties sur l'île puisque la côte ouest est plus arrosée que la côte est. Zanzibar est à l'écart des itinéraires habituels des cyclones, cette précision est essentielle pour la compréhension et l'organisation de l'espace touristique du sudouest de l'océan Indien car les îles des Mascareignes et la côte est de Madagascar sont souvent perturbées par la saison cyclonique de décembre à mars.

Rythmes des climats, rythmes des entrées touristiques à Zanzibar (d'après J.-L. Staszak).

\begin{tabular}{|l|l|l|l|l|}
\hline SAISON & $\begin{array}{l}\text { Petite saison } \\
\text { sèche }\end{array}$ & $\begin{array}{l}\text { Grande saison des } \\
\text { pluies }\end{array}$ & Grande saison sèche & $\begin{array}{l}\text { Petite saison des } \\
\text { pluies }\end{array}$ \\
\hline Mois & $\begin{array}{l}\text { Janvier- } \\
\text { Février }\end{array}$ & Mars-Avril & Juin-Septembre & Octobre-Décembre \\
\hline Circulation & $\begin{array}{l}\text { N.E. } \\
\text { (mousson) }\end{array}$ & S.E. (alizé) & S.E. (alizé) & N.E. (mouss on) \\
\hline $\begin{array}{l}\text { Précipitations } \\
(\mathrm{mm})\end{array}$ & $50-80$ & $220-400$ & $30-60$ & $90-200$ \\
\hline $\begin{array}{l}\text { Températures } \\
\text { (c })\end{array}$ & $27-29$ & $26-27$ & $23-25$ & $25-26$ \\
\hline $\begin{array}{l}\text { Nombre de } \\
\text { touristes } \\
\text { (moy./mois) }\end{array}$ & 7300 & 4850 & 7870 & 8560 \\
\hline
\end{tabular}

Une rapide analyse géomorphologique peut expliquer, dans une certaine mesure, la localisation et la prédominance des hôtels sur la côte est. Ce littoral se compose d'un plateau corallien bas, propice au développement balnéaire, avec de longues plages de sable blanc rectilignes sur plusieurs kilomètres (de Matemwe à Jambiani). Seule la baie de Chwaba (carte ${ }^{\circ} 2$ ), domaine de la mangrove, brise la linéarité de ce littoral. Les sols de cette côte sont beaucoup moins riches et fertiles qu'ailleurs. L'histoire du peuplement et des activités de Zanzibar montre que cette côte a toujours été, avant l'arrivée des touristes, périphérique voire délaissée. Les densités de population y sont faibles $\left(60\right.$ hab. $\left./ \mathrm{km}^{2}\right)$ comparées à la moyenne de l'île $\left(275\right.$ hab. $\left./ \mathrm{km}^{2}\right)$. Quelques petits villages de pêcheurs apparaissent aujourd'hui noyés entre les villages-clubs. À l'opposé, la côte ouest est constituée d'une table calcaire du 
miocène donnant un relief plus marqué à son littoral (de Makoba au nord à Kizimkazi au sud). Les plages sont de médiocre qualité, ce qui justifie la présence d'un seul et unique hôtel de plage.

\subsection{Un héritage culturel et historique unique}

Zanzibar possède une culture très riche où diverses influences se sont mêlées pour former une société unique en son genre. Pendant plus de 150 ans (15031668), les Portugais se sont appropriés les lieux, mais aucune trace de ces premiers chrétiens ne subsiste sur l'île. Par contre, les Arabes d'Oman, nouveaux maîtres de Zanzibar, après avoir chassé les Portugais (repliés plus au sud, au Mozambique) ont largement marqué les paysages zanzibaris. Aujourd'hui, le Fort arabe (ou Vieux fort), sur le front de mer de la vieille ville, est l'un des vestiges les plus visités; il abrite le Zanzibar Cultural Center. À partir de 1698, le sultan d'Oman dirige Zanzibar, Lamu et Mombassa. C'est le début de la triste période de l'esclavage. Dès 1832, le sultan d'Oman transfère sa capitale de Mascate à Zanzibar, c'est la période faste de l'île. La fin du $\mathrm{XIX}^{\mathrm{e}}$ siècle est prospère, l'île est le premier producteur mondial du clou de girofle. Zanzibar-ville est la porte d'entrée de l'Afrique de l'Est par la mer et le point de départ de nombreuses expéditions en Afrique continentale (Burton, Speke, Grant et Livingstone).

Stonetown forme un labyrinthe découpé par un dédale de ruelles étroites bordées de maisons de corail ; la vieille ville constitue un véritable musée. Balcons, tourelles, portes sculptées sont autant de témoignages de l'occupation arabe des $\mathrm{XVIII}^{\mathrm{e}}$ et $\mathrm{XIX}^{\mathrm{e}}$ siècles. Les somptueuses façades de la vieille ville sont en partie rénovées et favorisent le tourisme culturel. Hors de la ville, les vestiges arabes sont d'un réel intérêt : les ruines du Maharubi Palace, à quatre kilomètres au nord, palais ayant appartenu au sultan Bargash ; les restes de Mtoni, demeure de la princesse Salme, ou encore les anciens bains perses de Kidichi construits en 1850 par le sultan Said. À partir de 1895, Zanzibar se tourne vers la Tanzanie continentale mais garde sa double appartenance aux mondes arabe et africain.

Aujourd'hui, Zanzibar est l'un des centres les plus cosmopolites de la côte d'Afrique orientale. Différentes traditions culturelles s'y sont rencontrées, donnant naissance à la culture swahilie. De cette culture est née une tradition urbaine composée de différents styles empruntés aux pays de l'océan Indien. 


\subsection{L'ouverture politique et économique : projets et réalités}

Le tourisme se développe dans un contexte de transformation politique, économique et sociale. Le tournant se fait en 1984 avec, dans un premier temps, un changement politique. Une nouvelle orientation s'imposait car le «socialisme» de J. Nyerere s'était soldé par un constat d'échec. Zanzibar était coupée du monde. La crise des prix du clou de girofle ajoutée à une faillite devenue chronique du système économique avaient conduit l'île au bord du chaos.

Depuis 1984, sous la direction des présidents Jumbe puis Mwinyi, le pays s'oriente vers une libéralisation politique et économique. L'Investment Act de 1986 constitue le cadre juridique de l'ouverture. Dans ce texte, le gouvernement de Zanzibar définit six axes prioritaires : l'agriculture, l'industrie les transports, la construction, les services et le tourisme. Pour développer ces priorités, des facilités juridiques et fiscales sont proposées : privatisation, appel aux investisseurs étrangers et pour inciter les zanzibaris de la diaspora à investir dans le pays.

Le pays tente d'attirer les capitaux en fournissant garanties et avantages. Zanzibar vise à développer une économie insulaire et à devenir le «HongKong africain ». Il s'agit de mettre en valeur l'insularité en tant qu'atout économique. Zanzibar deviendrait un petit monde à part grâce à ses zones franches. Quatorze ans après, nous constatons que cette orientation était utopique. L'agriculture et l'élevage ainsi que l'industrie ont réalisé peu de progrès. Cette volonté d'ouverture a profité au secteur tertiaire avec particulièrement le tourisme qui a monopolisé $83 \%$ de l'investissement étranger contre $9 \%$ pour les transports, $3 \%$ pour l'agriculture et la pêche, $3 \%$ pour l'industrie et $2 \%$ pour les affaires et services. Les blocages dans les autres secteurs étaient trop marqués, seule cette branche de l'économie a semblé rassurer les investisseurs étrangers.

La part des capitaux étrangers dans l'hôtellerie est majoritaire dans l'île. Là encore, nous pouvons noter une dichotomie bien marquée entre le littoral est, où les capitaux italiens, sud-africains, allemands sont supérieur à $65 \%$, et la partie occidentale (Stonetown et Zanzibar-ville) où les zanzibaris ont largement investi depuis une demi-douzaine d'années (carte $\left.\mathrm{n}^{\circ} 2\right)$.

\subsection{Le défi des transports}

$\mathrm{Au}$ début des années quatre-vingts, Zanzibar était peu reliée avec le monde. L'évolution des transports va radicalement modifier son caractère insulaire. 
Le temps ou seuls les boutres (dhow) reliaient l'île au continent n'est pas si lointain. Jusqu'en 1996, la majorité des touristes arrivaient à Zanzibar en bateau (hydroglisseurs) de Dar-es-salaam ou de Mombassa. Sur les 69000 entrées touristiques de 1996, 35500 sont arrivées par le port de Stonetown et 33500 à l'aéroport de Kisauni. Le tournant s'opère en 1997, puisque $54 \%$ des touristes sont arrivés par voie aérienne. L'évolution semble s'être confirmée en 1998. Aujourd'hui, l'aéroport de Zanzibar permet à trois longs courriers de se poser. Ce sont les vols de Milan (un vol par semaine), de Qatar (un vol par semaine) et de Mascate-Oman- (quatre vols par semaine). Les motivations qui sont à l'origine de l'ouverture de ces vols sont différentes : touristiques pour le vol de Milan, économiques et politiques pour les vols de Qatar et Mascate (Oman).

La Tanzanie continentale (Dar-es-salaam et Arusha) et le Kenya (Nairobi et Mombassa) sont aujourd'hui très bien reliés à Zanzibar par Air Tanzania et Kenya Airways avec quatre vols hebdomadaires. Des vols locaux et quotidiens rapprochent également Zanzibar de Pemba.

Quatre compagnies maritimes assurent la liaison avec Dar-es-salaam plusieurs fois par jour (en une heure et quart) avec des hydroglisseurs pouvant contenir jusqu'à 120 passagers.

Boutres et hydroglisseurs se croisent aujourd'hui au large de Zanzibar et symbolisent les rapides mutations de l'île. Avec cette ouverture sur le monde, Zanzibar perd sans aucun doute de plus en plus de son insularité, mais va-telle gagner en îléité ?

\subsection{Une conjoncture touristique régionale favorable}

Le contexte actuel du tourisme dans la région apparaît favorable à Zanzibar. La proximité du Kenya, premier pôle touristique de l'Afrique de l'Est, a d'abord été une opportunité pour la Tanzanie continentale. Les réserves kenyanes sont toujours les plus visitées mais semblent victimes depuis quelques années d'un transfert d'un grand nombre de touristes vers les grands parcs nationaux du nord de la Tanzanie (parcs nationaux d'Arusha, de Tarangire, du lac Manyara, du Serengeti) ou de la région protégée du Ngorongoro. Grâce à ses lacs historiques (lac Tanganyika, lac Victoria) ou ses montagnes et volcans (Kilimandjaro), la Tanzanie offre davantage d'espaces diversifiés et « sauvages ».

Zanzibar profite de ce glissement et récupère une part non négligeable de visiteurs. Car, à la différence du Kenya, le littoral tanzanien a peu de poten- 
tialités balnéaires, il n'a pas l'équivalent de Mombassa ou de Malindi. Zanzibar n'a actuellement aucun concurrent «balnéaire» en Tanzanie. Pemba, malgré la beauté de ses plages et de son lagon, ne peut inquiéter l'essor de sa voisine du sud ; sa capacité hôtelière atteint 10 pensions avec un total de 69 chambres.

Zanzibar bénéficie de la crise que traverse aujourd'hui le tourisme balnéaire au Kenya (Lamu, Mombassa et Malindi). Ces trois stations balnéaires sont de plus en plus délaissées par le tourisme international en raison d'exactions commises par des bandits sévissant sur cette partie du littoral.

L'île de Mafia (carte $\mathrm{n}^{\circ}$ 1), beaucoup plus petite avec seulement $500 \mathrm{~km}^{2}$, pourrait ébranler la situation de monopole «balnéaire » de Zanzibar dans les prochaines années car le sud de la Tanzanie est, depuis peu, découvert par le tourisme international (région du Selous). L'idée selon laquelle la plupart des îles sont tributaires d'un continent se confirme avec cet exemple. Il faut aussi se replacer dans une région plus vaste et par conséquent changer d'échelle pour mieux saisir et appréhender l'évolution du tourisme de cet espace insulaire. Le sud de la Tanzanie propose des safaris centrés sur le Selous et des séjours balnéaires sur l'île de Mafia, recréant ainsi la même organisation spatiale du tourisme du nord du pays. L'île la plus au sud de la Tanzanie insulaire commence à attirer une partie de la clientèle italienne (des structures hôtelières italiennes sont en cours de réalisation).

\section{Une nouvelle organisation de l'espace née du développement du tourisme}

Les touristes occupent et parcourent inégalement l'espace à Zanzibar. Une nouvelle organisation du territoire est née d'une mise en tourisme différencié de l'île. Les paysages zanzibaris ont subi plus de transformations en dix ans qu'au cours du siècle passé. Nos critères d'analyse s'appuieront sur l'intensité géographique des phénomènes touristiques à partir des données quantitatives et qualitatives disponibles. Une étude des lieux touristiques de Zanzibar nous renvoie à deux grands types d'espaces : le premier, polyvalent et ouvert, le second, enclavé plus ou moins fermé. Cette approche typologique peut-elle déboucher sur un modèle reconnaissable à d'autres espaces touristiques micro-insulaires de la zone intertropicale ? 


\subsection{Stonetown : \\ un espace touristique urbain littoral non balnéaire}

«Tout espace est porteur, a priori, d'activités touristiques, mais il apparaît nécessaire d'en fixer les caractères et limites géographiques » ${ }^{6}$. A Zanzibar, on peut distinguer un type d'espace touristique partagé avec d'autres types d'activités dont la complémentarité est souvent indispensable à la production et à la consommation touristique. C'est le cas de la vieille ville de pierre (Stonetown). Le tourisme, même s'il ne représente pas les principales activités et formes d'occupation de l'espace, est néanmoins créateur d'espaces.

Les activités balnéaires sont marginales, les plages sont rares et de médiocre qualité et peu fréquentées par les touristes qui préfèrent le charme du centre ancien. C'est l'exemple-type de la station touristique urbaine littorale non balnéaire.

La dynamique de l'espace touristique de Stonetown est bipolaire puisque coexistent, d'une part, un front de mer et, d'autre part, un centre ancien :

- Le front de mer est aujourd'hui en cours de rénovation et ne possède que deux hôtels de classe internationale : le Serena (54 chambres - capitaux italiens) et le Tembo (34 chambres - capitaux locaux) et deux restaurants, le Blue (capitaux sud-africains) et le Seaview (capitaux locaux). Ce front de mer a de réelles potentialités, outre le ballet incessant des boutres sans âge, ce site abrite des merveilles architecturales parmi les plus remarquables de la côte Est africaine. Le fort Arabe, le Palais du Peuple (Beit-al-Sahel), ancien palais des sultans, la maison ou le palais des merveilles, l'ex-consulat britannique, le centre culturel Aga Khan, vaste et ancienne demeure du XIX ${ }^{\mathrm{e}}$ siècle, et surtout les jardins Jamituri, situés près du fort Arabe. C'est la promenade favorite des autochtones, surtout le dimanche soir où l'animation bat son plein avec des dizaines de vendeurs de brochettes et autres marchands de souvenirs (venant spécialement de Dar-es-Salaam). Cette double animation africaine et orientale séduit plus d'un visiteur malgré l'aspect encore délabré et peu structuré de ce site qui sera, à n'en pas douter, un des hauts lieux du tourisme dans la région. Le front de mer de Stonetown peut être considéré comme un espace touristique en attente mais non périphérique.

- La vieille ville, adossée au front de mer, a subi d'importantes taunsformations. La politique à l'égard de Stonetown a profondément changé pendant les dix dernières années, elle s'est accompagnée d'une politique de libéralisation du commerce et de développement touristique. Plus de 125 maisons appartenant au gouvernement ont été vendues et la proportion d'immeubles aux mains du gouvernement a baissé d'environ $40 \%$. De plus, le secteur de la 
construction est en plein essor et l'industrie du bâtiment affiche une croissance de $135 \%$. La vieille ville loge plus de 15000 personnes, abrite la plupart des bureaux du gouvernement, possède $40 \%$ des bâtiments scolaires et concentre les différentes religions du monde avec 48 mosquées, 4 temples indiens et 2 cathédrales ${ }^{7}$. Si Stonetown est loin d'être un lieu spécifiquement touristique, néanmoins certains quartiers semblent, en haute saison, ne vivre que par le tourisme tant les boutiques de souvenirs, hôtels, restaurants et bars sont concentrés. C'est ici que de belles demeures du XIX ${ }^{\mathrm{e}}$ siècle ont été rénovées et transformées avec beaucoup de réussite en hôtels de charme : Emerson' House dans le quartier de Mkunazini, le Baghani motel, le New Shangani hotel.

La vieille ville et son front de mer sont bien un espace touristique polyvalent et ouvert. Cette station est dite endotrope car sa mise en valeur repose sur l'utilisation touristique d'éléments internes à son tissu bâti (monuments, demeures, portes et musées). Ce type de station littorale non balnéaire est unique dans le milieu insulaire du sud-ouest de l'océan Indien. Les Seychelles, Mayotte, les îles des Mascareignes ne possèdent pas de tel site. Lamu (Kenya) et Moroni (Comores) s'en rapprochent mais sans posséder les mêmes richesses architecturales (Lamu) ni la même intensité touristique (Moroni).

\subsection{La côte est : un espace touristique enclavé et fermé}

Depuis 1990, la côte Est est un espace convoité et investi par le tourisme international. Le paysage littoral, de l'îlot Mnemba jusqu'à Jambiani (carte $\mathrm{n}^{\circ}$ 2), a été en grande partie bouleversé. Le tourisme est ici dévoreur et marqueur de territoires. Le littoral Est était marginal voire périphérique par rapport à la côte occidentale ; seuls quelques petits villages de pêcheursagriculteurs lui donnaient un peu de vie. À partir des années quatre-vingt dix, les changements sont soudains et brutaux. Parler ici de touristification de l'espace ne paraît pas usurpé. Un hôtel se positionne en moyenne tous les 800 mètres sur cet étroit liseré côtier. Ces « kystes » touristiques se sont intercalés entre les petits villages de pêcheurs, créant un paysage nouveau et atypique sur ce ruban littoral long de 45 kilomètres et large de moins de 300 mètres. Quel meilleur exemple de mise en tourisme des lieux !

À part les guest-houses de la première génération, la structure hôtelière est le village-club. Ces villages à seule vocation balnéaire et sportive sont intégrés et fermés. L'organisation est là aussi bipolaire : la plage et son lagon d'un côté et l'espace d'animation et d'hébergement de l'autre. Le bâti de ces structures hôtelières, avec tous les services nécessaires, contraste avec le 
dénuement et l'extrême pauvreté des villages de pêcheurs. Ces hôtels sont sans liaisons directes avec l'environnement social et culturel des villageois. Pas d'échanges économiques avec ce milieu d'accueil puisque les produits alimentaires proviennent de Dar-es-salaam. Nous sommes ici dans une logique de l'enclavement. Le touriste vit en vase clos, en marge de la vie ordinaire. Le village-club est une île dans une île. "C'est, au fond, un lieu de nulle part, un lieu universel, on se déplace mais on n'a pas bougé. ${ }^{8}$.

L'exemple de Zanzibar nous invite à réfléchir sur la fréquentation des lieux d'un espace micro-insulaire où cohabitent deux populations touristiques bien distinctes, où se juxtaposent deux systèmes.

L'un est replié dans sa coquille hôtelière et jouit des atouts naturels de l'insularité tropicale avec le cliché stéréotypé des trois « $\mathrm{S} »:$ sea, sun, sand. Ces touristes sont, bien sûr, centrés sur les littoraux nord et est. Stonetown ne représente guère qu'une brève parenthèse d'une journée dans leur séjour. Cette excursion est l'occasion pour eux de se consacrer à l'achat de souvenirs dans les nombreuses boutiques de la vieille ville. L'aspect culturel est loin d'être leur motivation première ; peu de temps est consacré à la visite du centre ancien et de son front de mer. C'est avant tout un tourisme sédentaire ; l'île est ici un espace plus occupé (côte est) que parcouru (Stonetown et l'intérieur de l'île).

L'autre est rarement en contact avec ces touristes sédentaires. Le centre de gravité des voyageurs est la vieille ville où ils séjournent. Zanzibar est pour eux un nom magique que leur imaginaire a transcendé.

«Le nom de Zanzibar, riche de connotations, renvoie à un imaginaire qui, pour ancien qu'il soit, est réactivé ou remobilisé par le tourisme. Zanzibar apparaît comme un absolu d'exotisme, un lieu d'aventure, c'est ce pays de rêve, cette utopie que le tourisme recherche » (J.-F. Staszak.)

Zanzibar est le lieu bien réel d'une territorialité imaginante. Les touristes sont à la recherche d'une ambiance exotique imprégnée de culture et d'histoire. C'est le type de tourisme culturel et itinérant centré sur un pôle urbain historique non balnéaire.

Cette présence simultanée de ces deux types d'espaces et de comportements peut surprendre sur une île aussi exiguë (moins de $1500 \mathrm{~km}^{2}$ ). L'espace zanzibari est bien marqué et différencié par le tourisme. Ce système, connu à l'intérieur des bassins touristiques de notre planète, l'est moins dans le sud-ouest de 1 'océan Indien car, en-dehors de Zanzibar, aucune île ne répond à ces critères. C'est un modèle rare et atypique dans les espaces micro-insulaires tropicaux. 
Le tourisme international s'est donc emparé des espaces littoraux qu'il a façonnés et modifiés à sa convenance. Nous avons montré un exemple de spatialisation touristique avec les phénomènes de linéarité, de complexité et de discontinuité. Mais cette intrusion brutale du tourisme n'est pas sans effets sur les hommes et leurs lieux de vie. Les conséquences souvent dénoncées du tourisme international sur les espaces micro-insulaires et principalement dans les économies sous-développées semblent se vérifier à Zanzibar, même s'il paraît un peu tôt pour dresser un bilan complet et précis. Les effets destructurants du tourisme sont néanmoins visibles et se traduisent par des impacts d'ordre socio-culturel et par des incidences sur l'environnement. La confrontation de deux cultures, celle des visiteurs au haut pouvoir d'achat et celle des modestes pêcheurs de la côte est, est évidente et saisit aussitôt l'observateur. N'oublions pas que nous sommes ici dans un des pays les moins avancés de la planète. "Ce phénomène conduira », rappelle G. Cazes, «à une modification superficielle, une altération sérieuse, une mutation générale ou une aliénation définitive des systèmes de valeurs traditionnelles ». Le processus d'acculturation est en marche et, depuis trois ans, nous constatons un glissement des emplois traditionnels vers les emplois dans l'hôtellerie. L'altération des valeurs traditionnelles s'opère donc depuis quelques années essentiellement sur le littoral est.

Il faudra être très attentif, dans un futur proche, aux incidences sur l'environnement et plus précisément sur la pollution des eaux (déversement d'eaux usées non épurées), sur la pollution des sites (dépôts d'ordures et de déchets solides), mais aussi aux effets de conflits et de concurrences par les espaces ${ }^{9}$. Il n'est plus à démonter que les écosystèmes littoraux sont très vulnérables.

Le tourisme pourrait être à Zanzibar un accélérateur du changement politique. Le pouvoir aujourd'hui mène une politique économique qui encourage le retour des arabes dans l'île. Il fragilise ainsi les fondements même du parti en place depuis 1964, le CCM (Parti de la révolution) et facilite l'ascension de son rival à Zanzibar, le CUF (Front civique uni) qui devrait très logiquement gagner les prochaines élections législatives de $2000^{10}$. Le CUF est constitué essentiellement de jeunes, frustrés et aigris par la crise et qui ressentent l'intrusion du tourisme comme une véritable agression culturelle. Le CUF est aidé financièrement par certaines organisations islamiques arabes aux thèses souvent plus extrêmes. On conçoit aisément que l'industrie du tourisme n'entre pas dans leurs problématiques de développement durable. Zanzibar a su résister, dans les siècles passés, à une histoire exceptionnellement mouvementée ; on peut douter qu'il sorte cette fois-ci indemne de la «déferlante » touristique. 


\section{Références}

AISNER P., PluSS C. (1983), La Ruée vers le soleil. Le tourisme à destination du Tiers-Monde, L'Harmattan, Paris.

BAILEY M. (1972), Les Relations extérieures de Zanzibar, Revue française d'études politiques africaines, 75 .

BONNIOL J.-L. (1987), « Micro-insularité et particularisme : approche comparée à partir de cas antillais et seychellois », Actes du colloque de Bordeaux, Iles tropicales : insularité, insularisme, collection « Îles et archipels », n ${ }^{\circ}$, CEGET, 1087.

CAZES G. (1975), Les Constantes spatiales du fait touristique littoral, TIGR.

CAZES G. (1983), Le Tourisme international dans le Tiers Monde : la problématique géographique, thèse d'État, Bordeaux.

CAZES G. (1989-1992), Les Nouvelles Colonies de vacances, L'Harmattan, 2 vol., Paris.

CAZES G. (1992), Fondements pour une géographie du tourisme et des loisirs, Bréal, Paris.

CAZES G., RAYNOUARD Y., L'Aménagement touristique, PUF, «Que sais-je $? »$, Paris.

CAZES G. (1999), «Les littoraux en proie au tourisme. Éléments de réflexion », Hérodote, 11093.

CODACCIONI-MEISTERSHEIM A. (1988), Insularité, insularisme, iléité. Quelques concepts pour l'étude des systèmes insulaires, Atelier MAB d'Ouessant.

Collectif (1989), «Iles et tourisme en milieux tropical et subtropical », CRET, Illes et archipels.

DeWAiLly J.-M., FlAMENT E. (1993), Géographie du tourisme et des loisirs, SEDES, Paris.

ESCOURROU P. (1993), Tourisme et environnement, SEDES-CDU, Paris.

GUÉBOURG J.-L. (1997), «Permanence et mutation des activités littorales dans les îles musulmanes swahilies de l'océan Indien », in Iles et littoraux tropicaux,. Actes des VIIèmes journées de géographie tropicale, Brest, 11-12-13 septembre, Ouest Éditions / Presses académiques.

LOZATO-GiotaRD J.-P. (1993), Géographie du tourisme, MassonGéographie.

Miossec J.-M. (1977), Un modèle de l'espace touristique, L'espace géographique.

MiosseC J.-M. (1998), «Le tourisme et les aménagements touristiques littoraux », in Géographie humaine des littoraux maritimes, SEDES-CNED. 
MOLES A. (1982), «Nissonologie ou sciences des îles », L'Espace géographique, $\mathrm{n}^{\circ} 4$.

PÉrON F. (1993), Des îles et des hommes. L'insUlarité aujourd'hui, Éd. de la Cité.

POLÉNYK M. (1992), «Zanzibar-Helgoland. Du mythe colonial à la Realpolitik», in L'Insularité. Thématique et représentations, L'Harmattan.

PRUNIER G. (1996), «Zanzibar à l'heure du culturalisme », Le Monde diplomatique.

Rossell P. (1984), Tourisme et Tiers-Monde : un mariage blanc, Ed. P.-M. Fabre, Lausanne.

SALEH M. (1996), «Zanzibar et le monde swahili », Afrique contemporaine, $\mathrm{n}^{\circ} 177$.

SANGUIN A.L. (sous la direction de) (1997), Vivre dans une île. Une géopolitique des inhumanités, L'Harmattan.

SHERIFF A. (1995), The History and conservation of Zanzibar stonetown, James Currey publishers, Londres.

SHERIFF A. (1998), «La vieille ville de pierre. Un héritage en transition », in Zanzibar aujourd'hui, sous la direction de Le Cour Grandmaison C. et Crozon A., Karthala.

Singaravelou, HuETZ de LeMPS C. (sous la direction de) (1986), Illes tropicales : insularité, insularisme, actes du colloque organisé à Bordeaux, collection « Îles et archipels », $n^{\circ} 8$, Bordeaux.

STASZAK J.-L. (1998), «L'ouverture économique et le tourisme : zones franches et villages-clubs », in Zanzibar aujourd'hui, Karthala.

VILlECROIX P. (1985), Le Mal-développement du tourisme en Haïti, thèse de doctorat de troisième cycle, Bordeaux III.

WACKERMANN G. (1994), Loisir et tourisme. Une internationalisation de l'espace, SEDES, Paris, coll. «Mobilité spatiale».

\section{Notes}

1. L'archipel de Zanzibar est situé à une faible distance du continent africain, $50 \mathrm{kms}$ pour Unguja et $36 \mathrm{kms}$ pour Pemba. Unguja compte 376000 habitants pour une superficie de $1464 \mathrm{~km}^{2}$, Pemba 265000 habitants pour $868 \mathrm{~km}^{2}$. Les densités sont donc importantes : $275 \mathrm{hab} . / \mathrm{km}^{2}$ pour l'archipel.

2. Pour plus de facilité, nous utiliserons Zanzibar pour désigner Unguja. Nous avons volontairement écarté de notre étude Pemba qui est très peu fréquentée par le tourisme international.

3. Cazes G. (1992), Fondements pour une géographie du tourisme et des loisirs, Bréal. 
4. Zanzibar fait partie géographiquement de l'espace du sud-ouest de l'océan Indien, l'archipel est à la même latitude que la plupart des îles des Seychelles ; mais Zanzibar n'entre pas dans les études de cet espace car toujours rattaché à la Tanzanie continentale.

5. Zanzibar a une capacité totale de 3828 lits : 1566 pour le littoral est, 1470 pour Stonetown, 584 pour Zanzibar-ville et 208 pour le littoral nord.

6. Cazes G. (1992), Tourisme et tiers-monde : un bilan controversé, L'Harmattan.

7. Sheriff A. (1998), «La vieille ville de pierre, un héritage en transition », in Zanzibar aujourd'hui, Karthala, IFRA.

8. Bruckner P. et Finkielkraut A. (1979), cité dans Tourisme et tiers-monde : un bilan controversé. Les nouvelles colonies de vacances? G. Cazes, L'Harmattan. 1992.

9. Dans le nord-est de l'île, une nouvelle agriculture se développe basée sur les algues marines et entre en compétition avec le tourisme pour le contrôle des plages.

10. Sans élections truquées, le CUF aurait déjà remporté les législatives d'octobre 1996. 